\title{
GW23-e1576 ASSOCIATION TISSUE KALLIKREIN WITH THE SEVERITY OF CORONARY ARTERY DISEASE
}

doi:10.1136/heartjnl-2012-302920v.2

Yu-yu Yao, Cong Fu, Gen-shan Ma, Yi Feng, Yu-yu Yao. Department of Cardiology, Zhongda Hospital, Medical School of Southeast University

Objectives The objective of this study was to examine the presence of tissue kallikrein (TK) in coronary artery disease (CAD) and its association with inflammation, intraplaque angiogenesis and plaque stability.

Methods Plasma TK concentrations were measured in 104 consecutive inpatients with newly diagnosed $\mathrm{CAD}$ and 33 in patients with normal coronary arteries who underwent first-time angiography for suspected $\mathrm{CAD}$, and their associations with angiographic indexes of the severity of $\mathrm{CAD}$ (number of diseased vessels and Gensini score) were estimated. Patients were divided into 3 groups according to the number of vessels affected. Vascular endothelial growth factor (VEGF), hs-CRP were measured in all subjects. Plaques were obtained from patients undergoing coronary endarterectomy, cross-sections underwent Movat's and Masson's trichrome staining, divided into stable and unstable plaque. TK, CD105, CD68 expression were assessed by immunohistochemistry.

Results Plasma TK was elevated in CAD. TK levels were significantly higher in patients with multi-vessel disease and acute obstruction of one major coronary artery (acute coronary syndrome, ACS) group than those single-vessel CAD, multivessel CAD and control $(p<0.01)$, but the concentration of hs-CRP was justly increased in ACS group, and there were no difference in VEGF among 4 groups. At third day after revascularisation, TK decreased from $3056 \pm 1246$ to 407 $\pm 350 \mathrm{pg} / \mathrm{ml}(\mathrm{p}<0.001)$. After adjustment for background risk factors, TK levels were an independent predictor of the severity of CAD. ROC curve analysis indicates that TK is the best indicator of ACS. Unstable plaque had more TK, macrophages and microvessel density compared with stable $(p<0.01)$. TK expression was mainly observed co- localisation in macrophages.

Conclusions Plasma TK levels are significantly higher in CAD and are correlated with the severity of the disease. Further clinical studies are needed to confirm the use of TK as a biomarker for the detection and extent of $\mathrm{CAD}$. 